\title{
Rotura hepática espontánea en el síndrome HELLP
}

\section{Sr. Director:}

Alrededor del 10\% de las gestantes que presentan preeclampsia pueden desarrollar un síndrome HELLP (anemia hemolítica, enzimas hepáticas aumentadas y bajo recuento de trombocitos). Presentamos el caso clínico de una paciente con síndrome HELLP que se complicó con rotura hepática espontánea y hemoperitoneo masivo.

Se trata de una gestante de 41 años, sin antecedentes de interés, sometida a cesárea urgente con histerectomía y extracción de feto viable por cuadro de preeclampsia grave, que ingresó en la unidad de cuidados intensivos para control postoperatorio. La analítica de ingreso mostró anemia (hemoglobina, 5 $\mathrm{g} / \mathrm{dl}$; hematocritos, 15\%), coagulopatía (actividad de protrombina, 35\%; tiempo de tromboplastina parcial activada, $63 \mathrm{~s})$, trombocitopenia $(35.000 / \mu \mathrm{l})$ y elevación de transaminasas (GOT, 3.000 U/l; GPT, 1.925 $\mathrm{U} / \mathrm{l})$.

Con sospecha de síndrome HELLP se inició transfusión de hemoderivados (concentrados de hematíes, trombocitos y plasma fresco congelado) y tratamiento con dexametasona (10 mg/12 h). En las siguientes $4 \mathrm{~h}$ la paciente presentó hipotensión arterial, taquicardia y oliguria a pesar de resucitación con fluidos y perfusión de vasoactivos. La exploración clínica reveló distensión abdominal progresiva. Se realizó tomografía computarizada de abdomen que mostró hematoma hepático masivo junto con abundante líquido libre intraabdominal (fig. 1). Se efectuó laparotomía exploradora, que constató hemoperitoneo $(3.000 \mathrm{ml})$ y hematoma subcapsular hepático. Se realizó packing hepático con 20 compresas. En el postoperatorio inmediato se apreció normalización hemodinámica y corrección de los parámetros de coagulación (actividad de protrombina del 50\%; tiempo de tromboplastina parcial activada, $30 \mathrm{~s}$; trombocitos, 60.000/ $\mu \mathrm{l}$ ). A las 48 h, la paciente volvió a quirófano para revisión, se extrajeron todas las compresas y se irrigó la cavi-

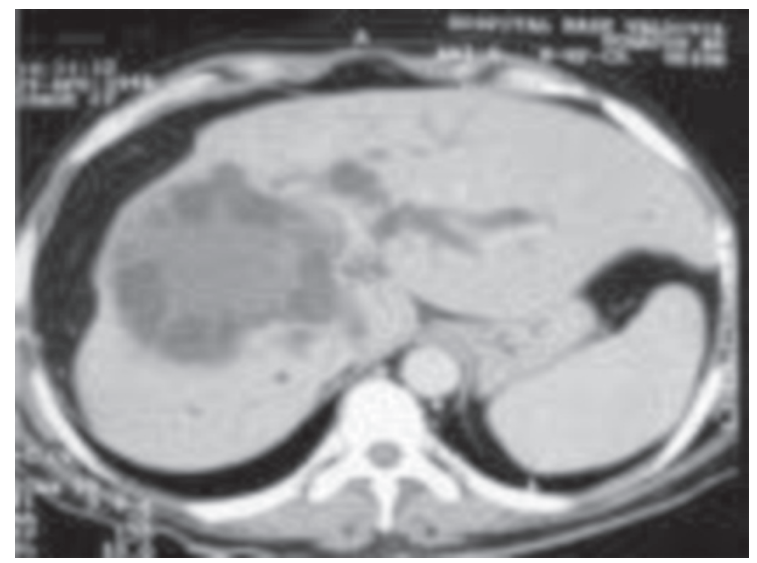

Figura 1. Hematoma subcapsular hepático. 
dad. No se apreció ningún punto de sangrado aunque el hígado presentaba un aspecto muy pálido y friable, por lo que se tomaron múltiples biopsias.

En las siguientes $24 \mathrm{~h}$ la analítica mostró signos de insuficiencia hepática con recurrencia de la coagulopatía (tiempo de tromboplastina parcial activada, 62 s, actividad de protrombina del $40 \%$ ) y aparición de hiperbilirrubinemia (12 mg/dl). El estudio histológico de las biopsias mostró necrosis difusa hepática con ausencia de focos de regeneración. Ante la irreversibilidad de la situación se decidió realizar trasplante hepático ortotópico urgente. Desafortunadamente, antes de realizar la transferencia al centro hospitalario de referencia, la paciente sufrió parada cardiorrespiratoria que no respondió a maniobras prolongadas de resucitación.

El hematoma subcapsular hepático es una complicación rara de la preeclampsia, que normalmente aparece en el marco de un síndrome HELLP. La incidencia general es de 1/45.000 a 1/225.000 nacimientos. La rotura hepática conlleva una mortalidad maternofetal del 50 y el $80 \%$, respectivamente ${ }^{1}$. La tomografía y la ecografía son las dos herramientas fundamentales de diagnóstico. En caso de rotura hepática contenida se puede adoptar una actitud expectante ${ }^{2,3}$. $\mathrm{Si}$ aparece hemoperitoneo masivo e inestabilidad hemodinámica, se recomienda tratamiento quirúrgico urgente. Lo más habitual es efectuar packing hepático evitando la resección del tejido hepático ${ }^{4}$. Algunos autores preconizan el uso de angiografía y embolización local. En casos de hemorragia exanguinante se puede recurrir a la hepatectomía y trasplante hepático de emergencia 5 .

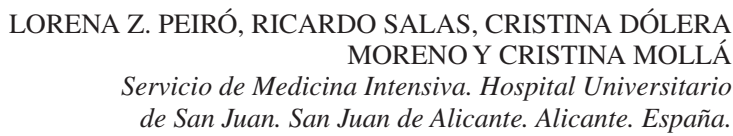

\section{BIBLIOGRAFÍA}

1. O'Brien JM, Barton JR. Controversies with the diagnosis and management of HELLP syndrome. Clin Obstet Gynecol. 2005;48:460-77

2. Magann EF, Martin JN. Twelve steps to optimal management of HELLP syndrome. Clin Obstet Gynecol. 1999;42:532-50.

3. Eser B, Guven M, Unal A, Coskun R, Altuntas F, Sungur M, et al. The role of plasma exchange in HELLP syndrome. Clin Appl Throm Hemost. 2005;11:211-7.

4. Vera EM, Pérez AC, Lattus OJ, Barrera CV, Campaña VG, Kattan MJ, et al. Rotura hepática asociada a preeclampsia severa y síndrome de HELLP: manejo y tratamiento con tamponamiento intraabdominal temporal de compresas. Rev Chil Obstet Ginecol. 2004;69:319-27.

5. Shames BD, Fernandez LA, Sollinger HW, Chin LT, D'Alessandro AM, Knechtle SJ, et al. Liver transplantation for HELLP syndrome. Liver Transpl. 2005;11:224-8. 